\title{
Global mRNA and Long Non-Coding RNA Expression in the Placenta and White Adipose Tissue of Mice Fed a High-Fat Diet During Pregnancy
}

\author{
Chen Huang ${ }^{a, b} \quad$ Bin-bin Huang ${ }^{a, b} \quad J i a n-m i n ~ N i u^{c} \quad Y^{2} Y_{u^{d}} \quad X i a o-y u n$ Qin $^{a}$ \\ Ya-li Yang ${ }^{a}$ Tian-xia Xiao ${ }^{a}$ Jie Chen ${ }^{a} \quad$ Li-rong Ren $^{d}$ Jian V. Zhanga \\ aLaboratory for Reproductive Health, Institute of Biomedicine and Biotechnology, Shenzhen Institute \\ of Advanced Technology, Chinese Academy of Sciences, Shenzhen, 'bhenzhen College of Advanced \\ Technology, University of Chinese Academy of Sciences, Shenzhen, 'Shenzhen Bao'an Traditional \\ Medicine Hospital Group, Shenzhen Maternity \& Child Healthcare Hospital Affiliated to Southern \\ Medical University, Shenzhen, 'Shenzhen Key Laboratory of Birth Defects, Shenzhen Bao'an Maternal \\ and Child Health Hospital, Shenzhen, China
}

\section{Key Words}

Adipose tissue $\bullet$ Gestational diabetes mellitus • Placenta • Microarray

\begin{abstract}
Background/Aims: Gestational diabetes mellitus (GDM) is a common complication of pregnancy, but the mechanisms underlying the disorders remain unclear. The study aimed to identify mRNA and long non-coding RNA (IncRNA) profiles in placenta and gonadal fat of pregnant mice fed a high-fat diet and to investigate the transcripts and pathways involved in the development of gestational diabetes mellitus. Methods: Deep and broad transcriptome profiling was performed to assess the expression of mRNAs and IncRNAs in placenta and gonadal fat from 3 mice fed an HFD and chow during pregnancy. Then, differentially expressed mRNAs and IncRNAs were validated by quantitative real-time PCR. The function of the differentially expressed mRNAs was determined by pathway and Gene Ontology (GO) analyses, and the physical or functional relationships between the IncRNAs and the corresponding mRNAs were determined. Results: Our study revealed that 82 mRNAs and 52 IncRNAs were differentially expressed in the placenta of mice fed an HFD during pregnancy, and 202 mRNAs and 120 IncRNAs were differentially expressed in gonadal fat. GO and Kyoto Encyclopedia of Genes and Genomes pathway analyses revealed differentially expressed mRNAs of placenta were closely related to extracellular matrix interactions, digestion, adhesion, and metabolism, whereas the differentially expressed mRNAs in adipose tissue were related to metabolic and insulin signalling pathways. The gene network demonstrated that

C. Huang, B.-b. Huang and J.-m. Niu contributed equally to this work.

\begin{tabular}{ll}
\hline Li-rong Ren & Laboratory for Reproductive Health, Institute of Biomedicine and Biotechnology \\
and Jian V. Zhang & Shenzhen Institute of Advance Technology, Shenzhen, Guangdong 518055 (China) \\
& Tel. (86) 0755-86392591, Fax (86) 0755-86585222, E-Mail 2554750789@qq.com; jian.zhang@siat.ac.cn
\end{tabular}
\end{abstract}


Actg2, Cnfn, Muc16, Serpina3k, NONMMUT068202, and NONMMUT068203, were the core of the network in placental tissue, and the genes Tkt, Acss2, and Elovl6 served as the core of the network in gonadal fat tissue. Conclusion: These newly identified key genes and pathways in mice might provide valuable information regarding the pathogenesis of GDM and might be used to improve early diagnosis, prevention, drug design, and clinical treatment.

(C) 2018 The Author(s)

Published by S. Karger AG, Basel

\section{Introduction}

The increasing population of overweight and obese in women were accompanied with growing incidence of gestational diabetes mellitus (GDM) in pregnancy women [1]. GDM is defined as glucose intolerance with onset or first recognition during pregnancy, which incidence is predicted to reach to $17.8 \%$ on worldwide [2, 3]. GDM was highly associated not only with acute increased risk for complications of pregnancy, such as hypertension, congenital abnormalities, and microsomia, and instrumental delivery [4-6], but also longterm metabolism complication disease for both mother and baby [7-9].

Many reports have investigated interaction between placenta and adipose tissue during pregnancy $[10,11]$. Adipose tissue secretes many adipokines, such as adiponectin, leptin, and tumour necrosis factor-alpha, regulate gestational processes like nutrient transportation, placental development, and foetal growth through it receptors on placenta $[12,13]$. Similarly, the hormones secreted from the placenta directly act on adipose tissue to suppress the sensitivity of insulin signals, inhibit glucose uptake, and promote fatty acid decomposition $[14,15]$. In GDM patients, many adipokines are dysregulated, indicating that adipose tissue plays an important role on the development of GDM $[16,17]$. Several reports have studied the expression profiles of coding and noncoding genes in the placenta of GDM patients [18-21], but the expression profile in adipose tissue has rarely been reported due to the limited availability and accessibility of human materials.

The incidence and prevalence of GDM have increased rapidly both locally and globally, but there are no reliable research tools for study of GDM $[3,8]$. The transitory nature of GDM leads to the study of its physiology difficultly [22]. Therefore, animal models was provided an attractive alternative for possible mechanisms of and therapeutics to GDM [5, 6]. Many groups have fed mice an HFD before or during pregnancy and evaluated insulin resistance, obesity, and foetal overgrowth, which are all well-established phenotypes in GDM [23-25].

In our study, we fed mice an HFD starting from pregnancy to mimic GDM. The HFD significantly elevated glucose and insulin levels, stimulated an imbalance of glucose metabolism, and increased the foetal weight. Both elevated glucose levels and increased foetal weight are well-established phenotypes in GDM. The placenta and gonadal fat from these mice were collected, and a global genome microarray analysis was performed to identify the differentially expressed mRNAs and long non-coding RNAs (lncRNAs), evaluate their relevant molecular functions and pathways, and explore the potential functions of these differentially expressed mRNAs and lncRNAs.

\section{Materials and Methods}

Animals and diets

C57BL/6 mice were purchased from the Laboratory Animal Centre, Institutes of Biomedicine and Health, Chinese Academy of Sciences. The animals were maintained at a constant temperature and humidity under a 12-h light-dark cycle. All procedures related to animal were carried out according to the guidelines of the Committee on the Use of Live Animals for Teaching and Research, Shenzhen Institutes of Advanced Technology, Chinese Academy of Sciences. Mating was confirmed by the presence of a vaginal plug the following morning, which was designated gestational day 0 . The mice were randomly divided into two groups and fed either a normal diet (standard chow) (6 mice) (10\% of energy from fat) or an HFD (5 mice) ( $45 \%$ of energy from fat from Research Diets, D12451) until gestational day 18. The body weight of the mice in both groups was monitored on gestational day 10 and day 15. On gestational day 17, intraperitoneal glucose tolerance test was performed as previously described [26]. Briefly, after fasting overnight (16 h), blood glucose levels were determined after an intragastric injection of $2 \mathrm{~g} / \mathrm{kg}$ glucose solution according to body weight. On gestational day 18, the mice were fasted for $6 \mathrm{~h}$, their serum was collected, and the 


\section{Cellular Physiology Cell Physiol Biochem 2018;50:2260-2271

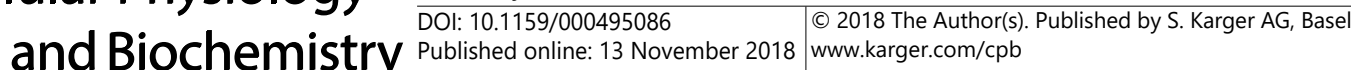

Huang et al.: Profiles of mRNA and IncRNA on Placenta and Fat

mice were euthanized via an intraperitoneal injection of tribromoethanol, followed by exsanguination. The placenta and gonadal fat were collected, snap-frozen in liquid nitrogen, and stored at $-80^{\circ} \mathrm{C}$ for further analysis. We randomly selected 3 placental and adipose tissue samples from each group for the lncRNA and mRNA detection array.

\section{Insulin assays}

On gestational day 18, the mice were fasted for $6 \mathrm{~h}$, and blood was collected from the orbital veins of the mice and clotted for $30 \mathrm{~min}$ at room temperature for serum collection. The total insulin level in serum was determined using a rat/mouse insulin enzyme-linked immunosorbent assay kit (EZRMI-13K, EMD Millipore, Burlington, MA, USA) according to the manufacturer's instructions.

\section{RNA processing and microarrays}

Total RNA was extracted using TRIzol reagent, qualified with $2 \%$ agarose, and quantified using an SMA 400 spectrophotometer. Sample labelling, microarray hybridization, and washing were performed according to the manufacturer's standard protocols. Fragmented cDNA was spotted onto a GeneChip mouse Gene 1.0 ST Array (Affymetrix, Santa Clara, CA, USA). Hybridization was performed according to the manufacturer's instructions. Signal scanning and analysis were performed using Affymetrix equipment (Affymetrix GeneChip Operating Software).

\section{Array data analysis and visualization}

The transcriptome profiles in the placenta $(n=6,3$ from HFD and 3 from chow) or gonadal fat $(n=$ 6, 3 from HFD and 3 from chow) were determined using the mouse GeneChip Mouse Gene 1.0 ST Array. The raw data (CEL files) were uploaded to the online working platform of Gene-Cloud of Biotechnology Information (https://www.gcbi.com.cn/gclab/html/index, Gminix Informatics Co., Ltd., Shanghai, China) for further analysis of the differences in mRNA and lncRNA profiles. Data were analysed with the significance analysis of microarray method to identify statistically significant genes by using Welch's $t$ test. For multiple comparison analysis, we computed the $q$ value to control the false discovery rate (FDR). A fold change (FC) $\geq 1.3$ (placenta) or FC $\geq 1.5$ (fat tissue) between the two groups and a $P$ value $<0.05$ was considered to indicate differentially expressed lncRNAs or mRNAs. Gene Cluster (version 3.0) was used to perform the hierarchical cluster analysis of differentially expressed mRNAs and lncRNAs. The functional clustering of the upregulated and downregulated genes was based on public databases according to the biological functions of their putative encoded proteins. The molecular pathways enriched by differentially expressed genes were obtained from the Gene Ontology (GO) and Kyoto Encyclopedia of Genes and Genomes (KEGG) pathways using the DAVID gene annotation tool (https://david.ncifcrf.gov/), and statistically overrepresented GO terms in the biological process and KEGG pathway were obtained by applying a Fisher's exact $P$-value cutoff $<0.01$ and correcting for multiple testing with the Benjamini FDR.

\section{Quantitative real-time PCR}

To validate the expression of dysregulated mRNAs and lncRNAs, placenta and gonadal fat were collected from chow-fed $(n=6)$ and HFD-fed $(n=6)$ mice during pregnancy. Total RNA from placenta or adipose tissue was extracted using the RNAiso Plus reagent (Takara, Shiga, Japan) and analysed by quantitative real-time PCR (qRT-PCR) according to the manufacturer's instructions (Revert RT Master and ReverTra qPCR RT Master Mix gDNA remover, Toyobo, Osaka, Japan). The primer sequences of mRNAs and lncRNAs are presented in Supplementary Table 1 (for all supplementary material see www.karger.com/ doi/10.1159/000495086). Gene expression levels were normalized to $18 \mathrm{~S}$ using the $\Delta \Delta \mathrm{CT}$ method, where CT is the cycle threshold. Melt curve analysis for each primer set revealed only one peak for each product. The sizes of the PCR products were confirmed by comparing the product size with a commercial ladder after agarose gel electrophoresis. All reactions were performed in triplicate.

\section{Statistical analysis}

Each experiment was repeated to produce three biological replicates. All of the statistical evaluations were performed using GraphPad Prism 7. The results are presented as the means \pm standard error of the mean. The differences between the two groups were determined by Student's $t$ test, where $P<0.05$ was considered statistically significant. 


\section{Results}

\section{Maternal and fetal characteristics}

After pregnancy, the mice were immediately divided into two groups and fed either chow or HFD. Their body weight was examined on gestational days 10 and 15, and no significant differences were found, as shown in Fig. 1A. However, fasting glucose was increased on gestational days 8 and 15 (Fig. 1B). The glucose tolerance test results demonstrated dramatic differences between the HFD group and chow group, as indicated by glucose concentration 15, 30, and $60 \mathrm{~min}$ after fasting $16 \mathrm{~h}$ (Fig. 1C). On gestational day 18, the level of insulin in serum was significantly increased in the mice fed HFD, as shown in Fig. 1D. The weights of foetus and placenta were determined, as shown in Fig. 1E and 1F. HFD significantly increased the level of fasting glucose, exacerbated glucose tolerance, and increased the foetal weight but did not affect the weight of the placenta.

\section{Identification of differentially expressed $m R N A$ and IncRNA profiles in placental tissue}

The genes differentially expressed in placenta of HFD and chow mice during pregnancy were analysed on day 18 using the GeneChip Mouse Genome Array. We scanned the mRNA probes of placental tissue and found that 82 mRNAs and 52 lncRNAs were differentially expressed with significant differences $(P<0.05)$, although these genes were not significantly differentially expressed according to their $q$ value because of the small group number (Suppl. Tables 2 and 3). Among these dysregulated mRNAs, 66 were upregulated and 16 were downregulated, and the differentially expressed lncRNAs included 28 unregulated and 25 downregulated genes. Hierarchical clustering showed distinguishable gene expression patterns between chow and HFD pregnant mice (Fig. 2A and B) and differentially expressed

Fig. 1. A. the body weight of mice fed chow or HFD was examined on gestational days 10 and 15 . B. the fast glucose in the chow- and HFD-fed groups was examined on gestational days $0,5,8,15$ and 18. C. Glucose tolerance test was performed on gestational day 17 . D. Insulin levels on gestational day 18. E-F. The fetus and placenta weights were measured after the mice were sacrificed on gestational day 18 . Values are presented as means \pm SEM. ${ }^{*} \mathrm{p}<0.05$, ${ }^{* *} \mathrm{p}<0.01,{ }^{* * * *} \mathrm{p}<0.001$ compared with chow group.
B

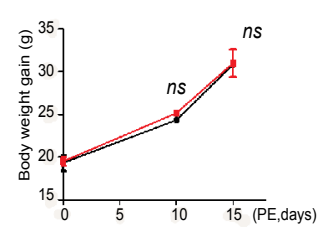

D

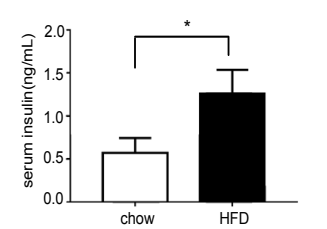

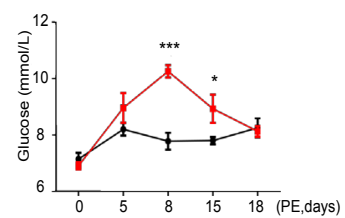

$E$

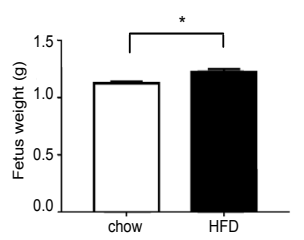

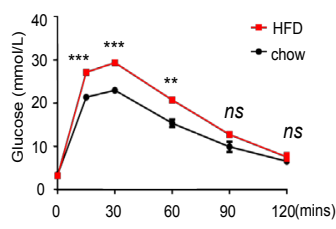

F

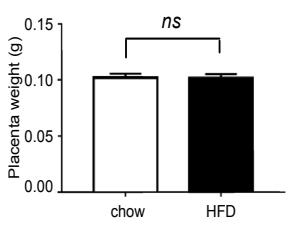

Fig. 2. Hierarchy of the differentially expressed mRNAs (A) and IncRNAs (B) in placenta between HFD and chow groups. The dysregulated mRNAs and $\operatorname{lncRNAs}(\mathrm{FC} \geq 2.0$ and $\mathrm{p}<0.05$ ) are indicated in Tables 1 and 2 .

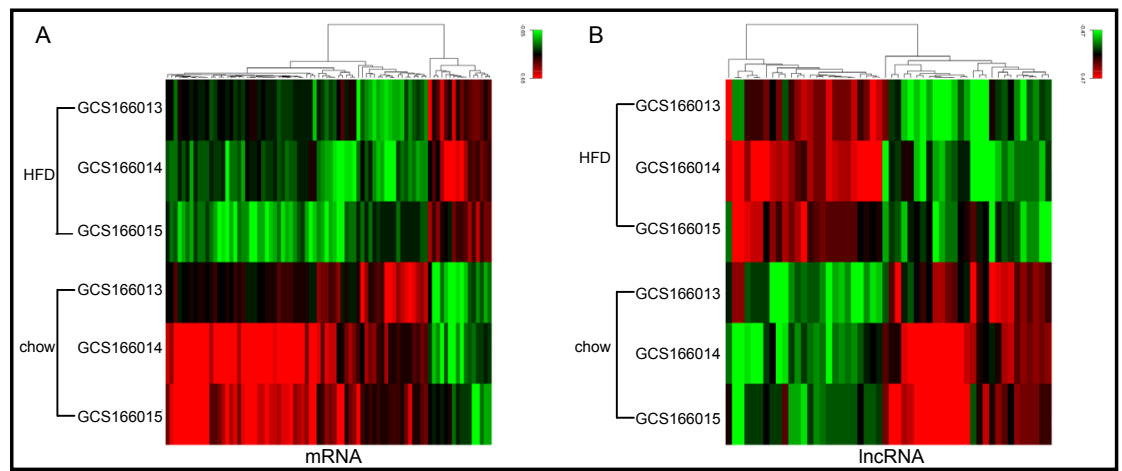




\section{Cellular Physiology Cell Physiol Biochem 2018;50:2260-2271

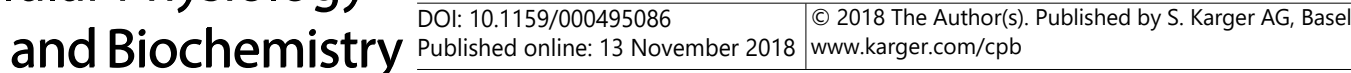

mRNAs and lncRNAs (FC $\geq 2.0$ and $P<0.05$ ) are shown in Tables 1 and 2 . These mRNAs included Prap1, Serpina3n, Ppbp, Guca2b, Actg2, Dpt, Muc16, Mal, and Prl7c1, and all of these, with the exception of Prl7c1, were upregulated. The differentially expressed lncRNAs included ONMMUT068204, NONMMUT068202, NONMMUT068206, and NONMMUT068203, and all of which were upregulated. In the placenta, Prap1 (FC $=4.266459)$ was the most significantly regulated mRNA, and NONMMUT068203 (FC $=5.057185$ ) was the most significantly regulated IncRNA.

\section{Differential $m R N A$ and IncRNA profiles in gonadal fat tissue}

The differentially expressed mRNA and lncRNA profiles in gonadal fat from chow and HFD pregnant mice were identified based on the criteria FC $\geq 1.5$ and $P \leq 0.05$. In total, 202 genes were differentially expressed, which included 61 upregulated and 141 downregulated mRNAs (Suppl. Table 4). Regarding the IncRNAs, 120 were differentially expressed and included 39 upregulated and 81 downregulated IncRNAs (Suppl. Table 5). Among them, 160 mRNAs and 75 lncRNAs were significantly changed according to the FDR tests. A hierarchical clustering is shown in Fig. 3A (mRNA) and 3B (lncRNA). The differently expressed genes ( $\mathrm{FC} \geq 2.5$ and $\mathrm{P} \leq 0.05$ ) included Elovl6, Acly, Gys2, igk, Gm6484, Slc15a5, Cldn22, Acss2, Mup3, Tlcd2, Orm3, Peg3, 1110059M19Rik, and Mest (Table 3). Among these genes, 11 were downregulated and 3 were upregulated. The lncRNAs included Gm11295, Gm3601, XR_141088, and XR_141492, which were all downregulated (Table 4). Elovl6 (FC = -4.39) was the most significantly regulated mRNA, and Gm11295 (FC $=-4.64)$ was the most significantly regulated lncRNA.

\section{Validation of dysregulated mRNA and IncRNA in placenta and gonadal fat tissue using} qRT-PCR analysis

To validate the aberrantly expressed mRNAs and lncRNAs, we selected several dysregulated mRNAs and lncRNAs to validate their expression by using qRT-PCR in chow $(n=$ $6)$ and HFD $(n=6)$ groups of pregnant mice. Fig. 4A and 4B shows the expression of 9 mRNAs (4 upregulated and 5 downregulated) and 8 lncRNAs ( 5 upregulated and 3 downregulated), and Fig. 4C and 4D shows the expression of 10 mRNAs ( 5 upregulated and 5 downregulated) and 8 IncRNAs ( 3 upregulated and 5 downregulated), most of which were consistent with the microarray data, demonstrated the reliability of the microarray data.

Co-expression network and potential pathway of mRNAs and lncRNAs in placenta and gonadal fat tissue

To determine the molecular function of the dysregulated mRNAs and lncRNAs in placental and gonadal fat from HFD-fed pregnant mice, we selected differentially expressed mRNAs and lncRNAs with $\mathrm{FC} \geq 1.5$ (placenta) or $\mathrm{FC} \geq 2.0$ (fat tissue) for KEGG pathway analysis. As shown in Fig. 5A, KEGG pathway analysis indicated that differentially expressed genes of placental tissue were more likely related to extracellular matrix (ECM) interactions,

Table 1. Differentially expressed mRNAs $(F C \geq 2.0)$ in placenta between HFD and chow groups

\begin{tabular}{lccccc}
\hline Probe Set ID & Gene Symbol & Accession Number & FC & p-value & Gene Feature \\
\hline TC0700002001.mm.1 & Prap1 & NM_009475 & 4.266459 & 0.030633 & up \\
TC1200001047.mm.1 & Serpina3n & NM_009252 & 4.073544 & 0.004643 & up \\
TC0500000842.mm.1 & Ppbp & NM_023785 & 3.883555 & 0.010269 & up \\
TC0400003433.mm.1 & Guca2b & NM_008191 & 2.15974 & 0.008104 & up \\
TC0600002574.mm.1 & Actg2 & NM_009610 & 2.104614 & 0.007735 & up \\
TC0100001510.mm.1 & Dpt & NM_019759 & 2.021738 & 0.031881 & up \\
TC0900001804.mm.1 & Muc16 & ENSMUST00000034653 & 2.014879 & 0.009142 & up \\
TC0200004563.mm.1 & Mal & NM_010762 & 2.004461 & 0.001975 & up \\
TC1300001699.mm.1 & Prl7c1 & NM_026206 & -2.46683 & 0.000519 & down \\
\hline
\end{tabular}

Table 2. Differentially expressed lncRNA $(F C \geq 2.0)$ in placenta between HFD and chow groups

\begin{tabular}{llccc}
\hline Probe Set ID & Accession Number & FC & p-value & Gene Feature \\
\hline TC0900001806.mm.1 & NONMMUT068203 & 5.057185 & 0.000174 & up \\
TC0900001808.mm.1 & NONMMUT068206 & 4.305119 & 0.000151 & up \\
TC0900001805.mm.1 & NONMMUT068202 & 3.73099 & 0.000308 & up \\
TC0900001807.mm.1 & NONMMUT068204 & 3.205073 & 0.000394 & up \\
\hline
\end{tabular}




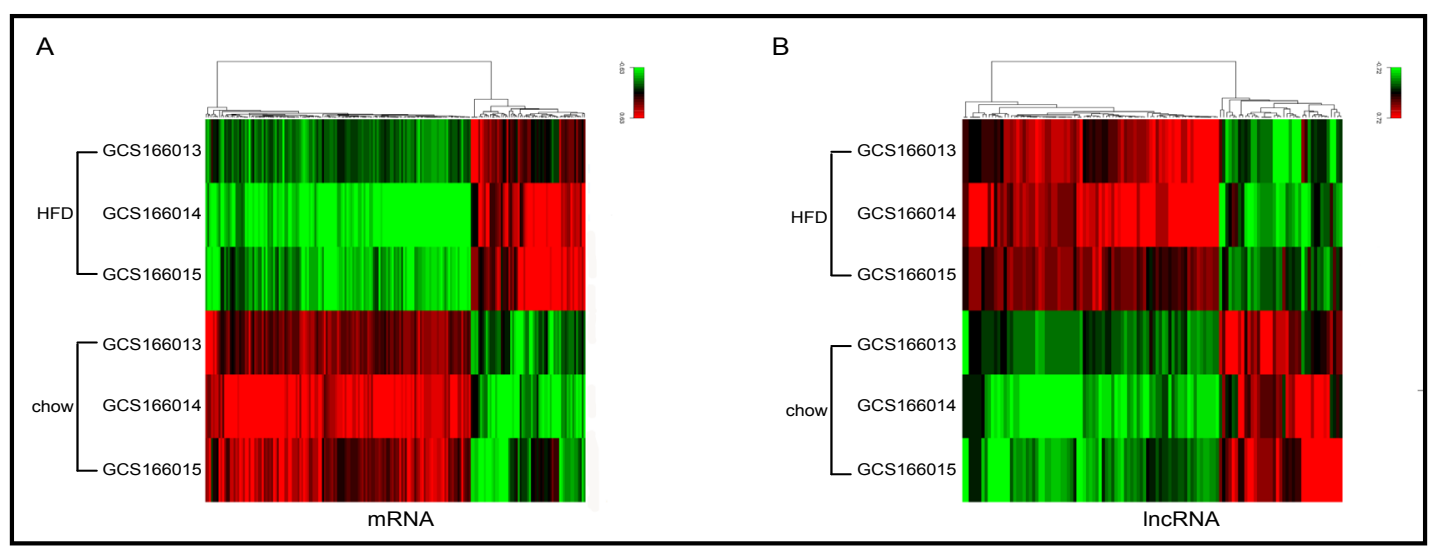

Fig. 3. Hierarchy of differentially expressed mRNAs (A) and lncRNAs (B) in gonadal fat tissue between HFD and chow groups during pregnancy. The dysregulated mRNAs and lncRNAs (FC $\geq 2.5)$ are indicated in Tables 3 and 4.

Table 3. Differentially expressed mRNAs $(F C \geq 2.5)$ in gonadal fat tissue between HFD and chow groups

\begin{tabular}{|c|c|c|c|c|c|}
\hline Probe Set ID & Gene Symbol & Accession Number & $\mathrm{FC}$ & p-value & Gene Feature \\
\hline TC0600000277.mm.1 & Mest & NM_001252293 & 2.990212 & 0.002082 & up \\
\hline TC0X00000414.mm.1 & 1110059M19Rik & NM_026841 & 2.600057 & 0.002323 & up \\
\hline TC0700002217.mm.1 & Peg3 & NM_008817 & 2.513965 & 0.000194 & up \\
\hline TC0400000720.mm.1 & Orm3 & NM_013623 & -2.50628 & 0.000768 & down \\
\hline TC1 100004270.mm.1 & Tlcd2 & NM_027249 & -2.55355 & 0.002577 & down \\
\hline TC0400004214.mm.1 & Mup3 & NM_001039544 & -2.62735 & 0.000296 & down \\
\hline TC0200002367.mm.1 & Acss 2 & NM_019811 & -2.67202 & 0.002595 & down \\
\hline TC0800000561.mm.1 & Cldn22 & NM_029383 & -2.68875 & 0.017636 & down \\
\hline TC0600003345.mm.1 & Slc15a5 & NM_177787 & -2.81876 & 0.006358 & down \\
\hline TC0900000205.mm.1 & Gm6484 & NM_001080940 & -2.98982 & 0.002505 & down \\
\hline TC0600003522.mm.1 & Igk & BC021781 & -3.043 & 0.008438 & down \\
\hline TC0600003374.mm.1 & Gys2 & NM_145572 & -3.10191 & 0.003174 & down \\
\hline TC1 $100003775 . \mathrm{mm} .1$ & Acly & NM_001199296 & -3.17097 & 0.002639 & down \\
\hline TC0300001306.mm.1 & Elovl6 & NM_130450 & -4.39397 & 0.004234 & down \\
\hline
\end{tabular}

Table 4. Differentially expressed lncRNAs $(F C \geq 2.5)$ in gonadal fat tissue between HFD and chow groups

\begin{tabular}{lccccc}
\hline Probe Set ID & Gene Symbol & Accession Number & FC & p-value & Gene Feature \\
\hline TC1300000199.mm.1 & Gm11295 & ENSMUST00000121379 & 4.648377 & 0.000467 & up \\
TC1100002049.mm.1 & & XR_141088 & 2.832155 & 0.000673 & up \\
TC1400001972.mm.1 & Gm3601 & XR_105655 & 2.756228 & 0.000142 & up \\
TC0300001634.mm.1 & & XR_141492 & 2.545996 & 0.000109 & up \\
\hline
\end{tabular}

digestion, adhesion (including ECM-receptor interactions, protein digestion and absorption, focal adhesion, the PI3K-Akt signalling pathway, amoebiasis, and proteoglycans in cancer), and metabolism (vascular smooth muscle contraction, phenylalanine metabolism, histidine metabolism, and beta-alanine metabolism). Similar to placental tissue, the differentially expressed genes of gonadal fat tissue were more enriched in the metabolic pathways (metabolic pathways, pyruvate metabolism, and fatty acid biosynthesis) and insulin signalling pathway (Fig. 5B). Suppl. Tables 6 (placenta) and 7 (fat tissue) indicate that the most enriched genes of placental tissue were upregulated, and those in gonadal fat tissue were downregulated.

Co-differentially regulated genes and involved pathways in placenta and gonadal fat tissue

The genes dysregulated in both placenta and gonadal fat tissues were selected for functional and pathway analyses. As indicated in Suppl. Table 8, 30 genes were dysregulated in placenta and gonadal fat tissue, which are involved in positive regulation of the protein kinase B signalling cascade, angiogenesis, cell adhesion, and cytoskeleton organization (Fig. 
6A). These genes were enriched in pathways like the complement and coagulation cascades, HIF-1 signalling pathway, haematopoietic cell lineage, and pentose phosphate pathway in Chagas disease (Fig. 6B).

mRNA and IncRNA network analyses in placenta and gonadal fat tissue

mRNA-lncRNA network analysis was performed to generate interaction networks containing the bio-relationships between all differentially expressed lncRNAs and mRNAs. Fig. 7A indicates mRNAs (Actg2, Cnfn, Muc16, Serpina3k, and Pgm5) and IncRNAs (NONMMUT068202 and NONMMUT068203) as the circular core of the pathway network, were more interacted with other genes, all of which genes in this network were upregulated. The network for gonadal fat is shown in Fig. 7B and indicates mRNAs Me1, Mup5, LOC100861909, Mup9, and Mup7 interacted more with other genes, and most of which were downregulated.

\section{Gene-gene interaction network in placenta and adipose tissue}

The significantly changed mRNAs and lncRNAs from placenta and gonadal fat were subjected to gene-gene interaction network analysis. The results from placental tissue (Fig. 8A) showed the genes (Actg2, Cnfn, Muc16, Serpina3k, Pgm5, and P4ha3) were directly or indirectly associated with Gfpt2, Ppbp, NONMMUT068202, NONMMUT068203 and

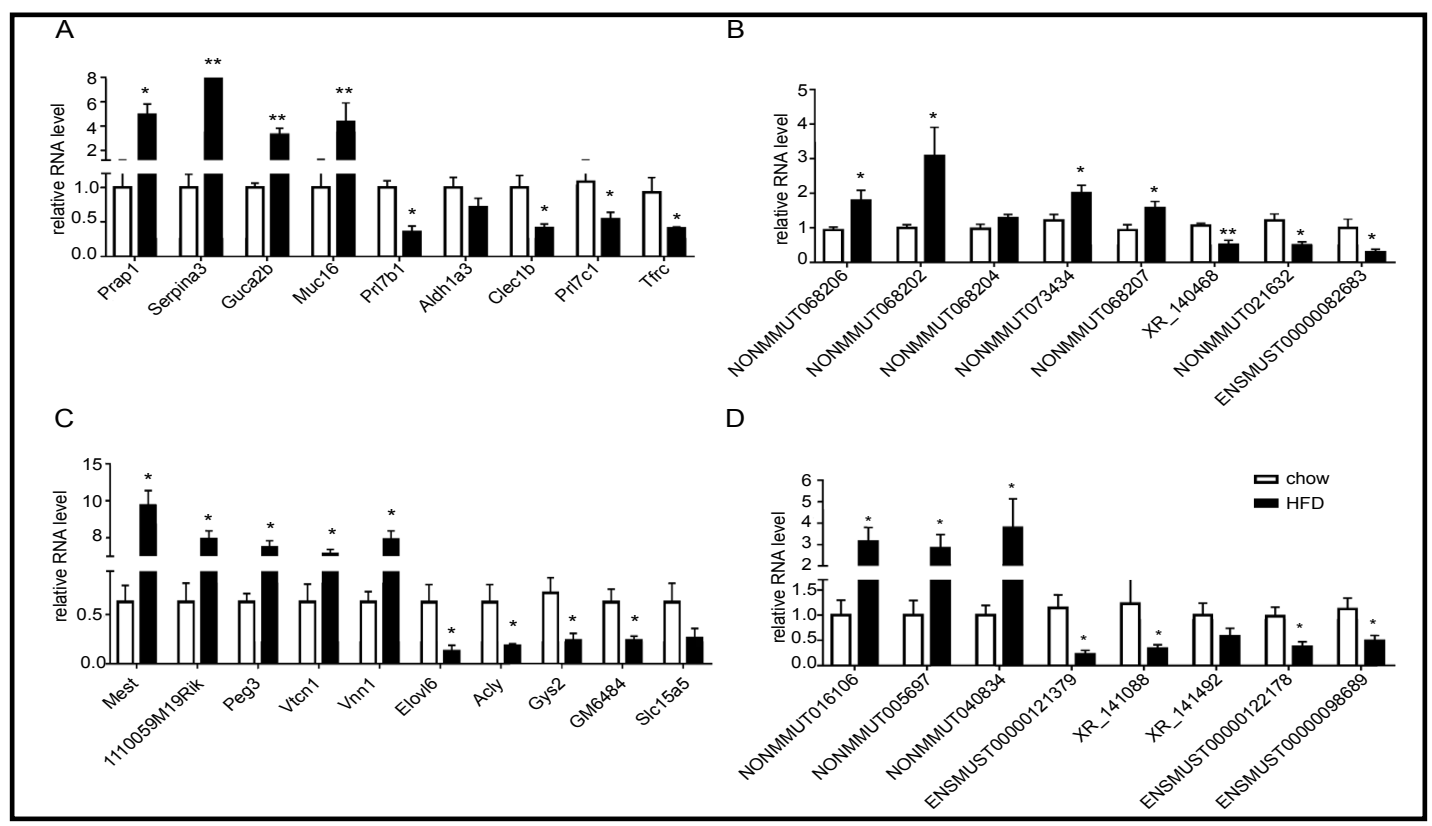

Fig. 4. Validation of significantly dysregulated mRNAs and IncRNAs of placenta and gonadal fat tissue by qRT-PCR. The Figure shows the up or down-regulated mRNAs and lncRNAs in placenta (A and B) and gonadal fat tissue $(C$ and $D)$ were consistent with the microarray data. Values are presented as means \pm SEM. ${ }^{*} \mathrm{p}<0.05,{ }^{* *} \mathrm{p}<0.01$, compared with chow group.

Fig. 5. Top 10 enriched pathways in placenta (A) and gonadal fat tissue (B) were calculated based on KEGG pathway analysis.

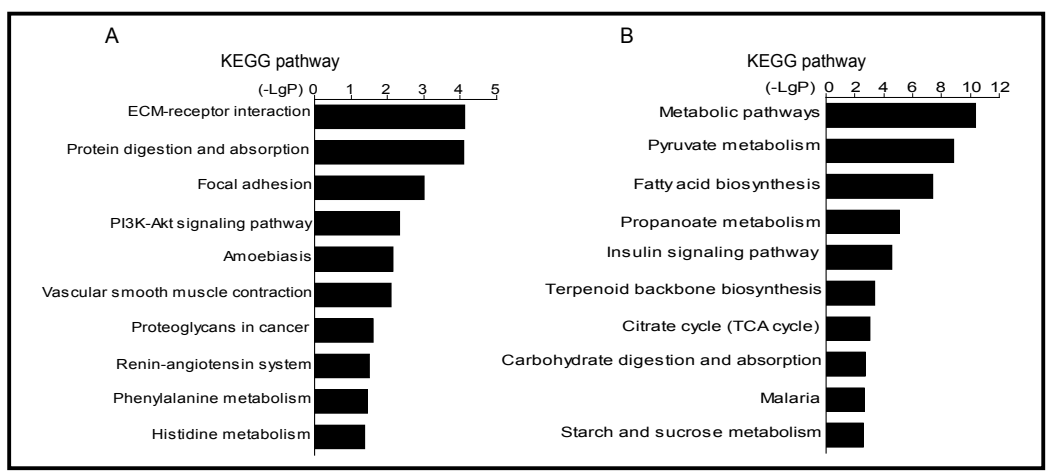


Fig. 6. Genes were co-differentially regulated in placenta and gonadal fat tissue and the associated functions (A) and KEGG pathways (B).
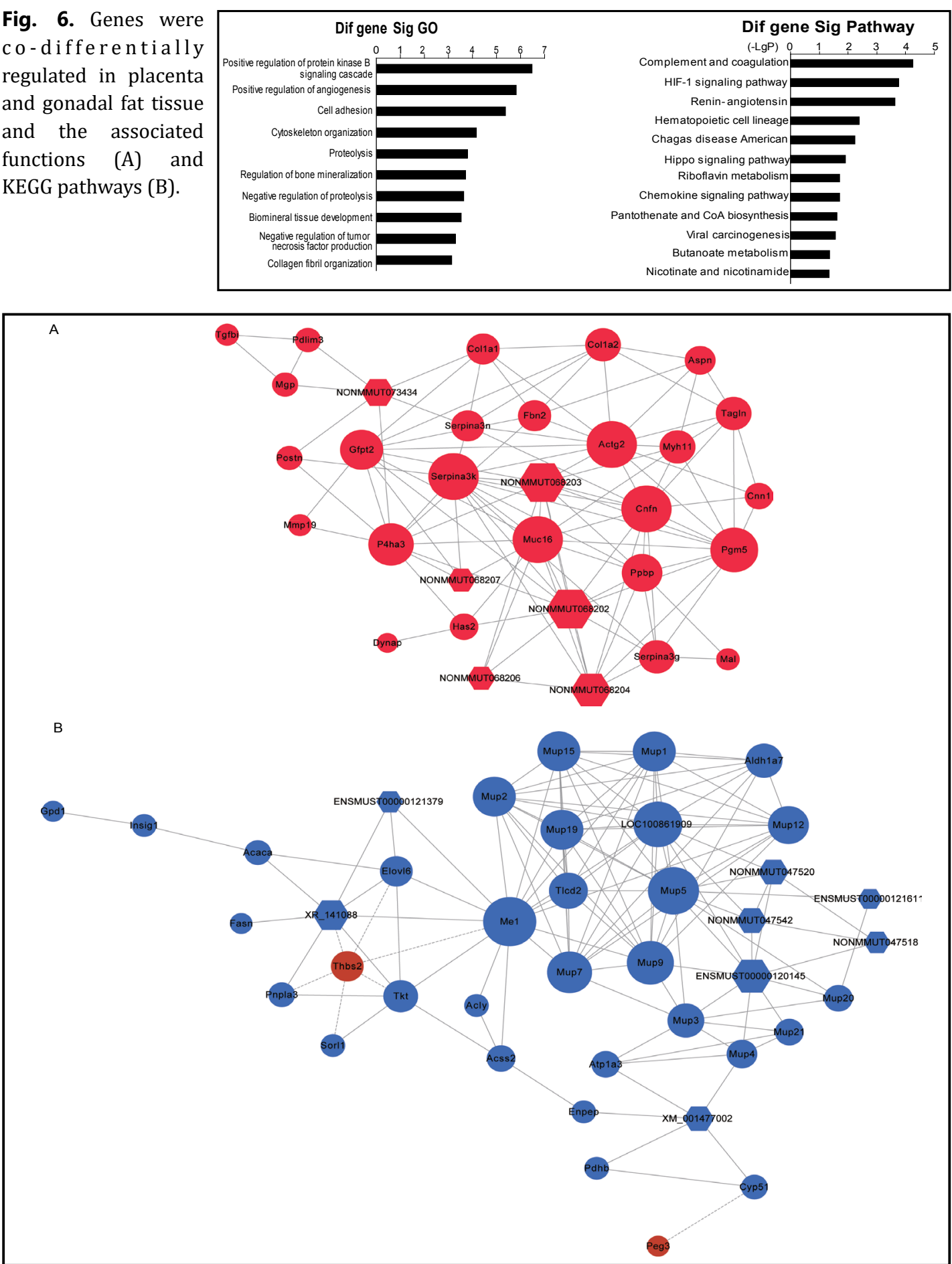

Fig. 7. Network of differentially expressed lncRNAs and target mRNAs according to p-value. (A) Placenta. (B) Gonadal fat tissue. The circles represent the genes, and the hexagon nodes represent the lncRNAs. The size of the circle or hexagon node represents the degree value; red represents the upregulated RNAs, and blue represented the downregulated RNAs.

NONMMUT068204, all of which were upregulated. The results from gonadal fat (Fig. 8B) indicated that these genes (Tkt, Acss2, and Elovl6) were strongly associated with Acaca, Enpep, Pnpla3, and Acly, all of which were downregulated.

\section{KARGER}




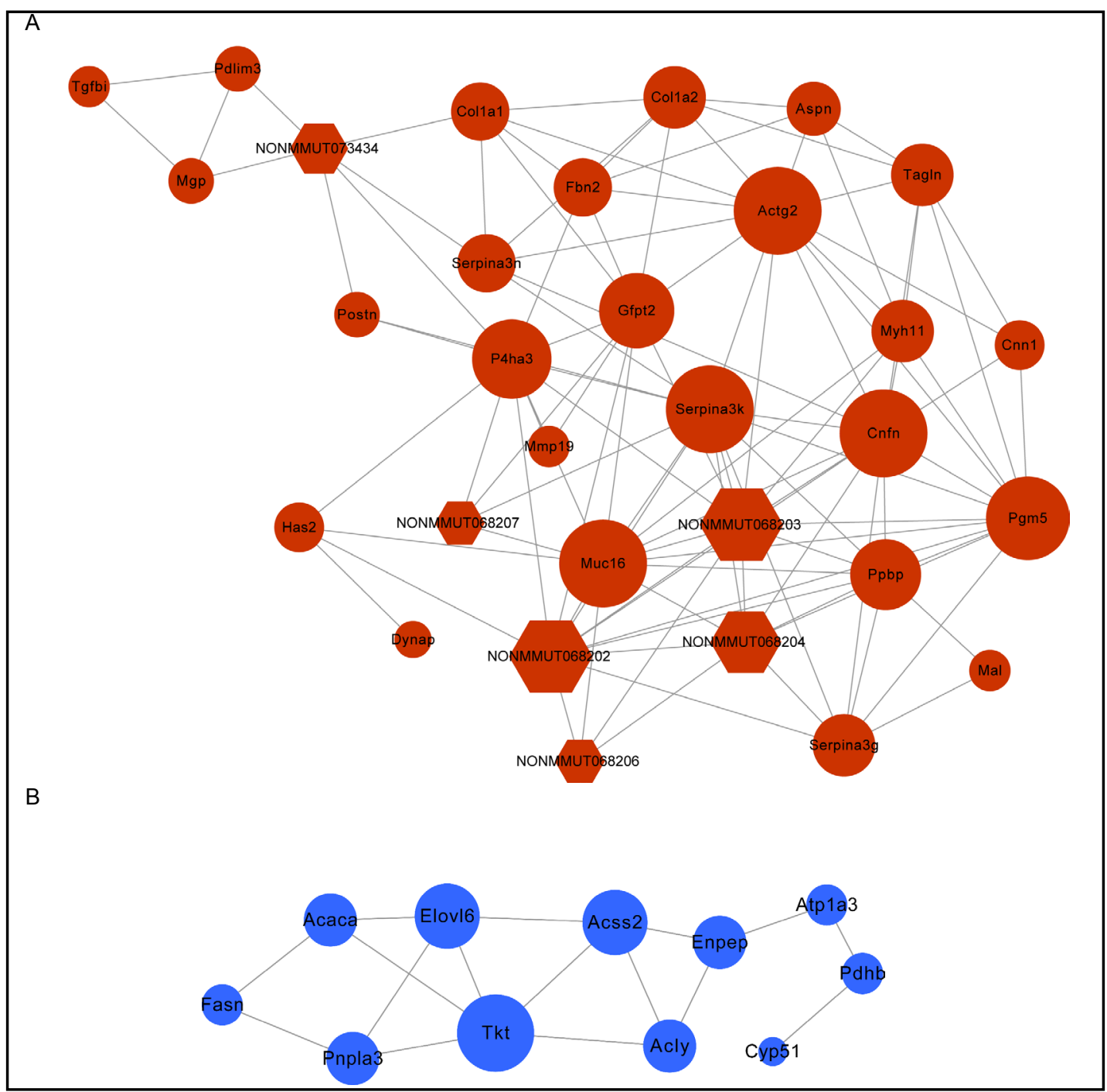

Fig. 8. Gene-gene interaction network in the placenta (A) and gonadal fat (B). The differentially expressed genes were analysed and identified by a gene co-expression network with a core algorithm. The cycle nodes represent genes, the size of the nodes represents the power of the interrelation among the nodes, and the edges between two nodes represent the interactions between genes. Red indicates the upregulation of genes, and blue indicates the down regulation of genes. The more edges of a gene, the more genes that are connecting to it and the more central role it plays within the network.

\section{Discussion}

GDM is considered a heterogeneous metabolic disorder that occurs during pregnancy and is a well-known risk factor for macrosomia [8]. Several studies using rodent models have been performed to identify the consequences of maternal obesity for foetal development $[25,27]$. This study fed pregnant mice with HFD to mimic human overnutrition during pregnancy. An HFD during pregnancy did not change body or placental tissue weight, but impaired glucose metabolism, as indicated by increased fasting glucose level, insulin level, exacerbated glucose tolerance, and significantly increased foetal weight, which are similar to symptoms of GDM in humans.

To identify the pathophysiological events that occurred during pregnancy in mice fed an HFD, placental and gonadal fat were collected to identify gene expression profiles. In placental tissue, we found 82 differentially expressed mRNAs and 52 dysregulated lncRNAs.

\section{KARGER}


Among them, $82 \%$ of mRNA and lncRNAs were upregulated, and only $18 \%$ of the RNAs were downregulated. Further analysis showed most of the genes with $\mathrm{FC} \geq 2.0$ were upregulated in the HFD group. The KEGG pathway and gene network analyses revealed most of the mRNAs and IncRNAs involved were also upregulated (Suppl. Table 1). In contrast to the results from placental tissue, 202 mRNAs and 120 lncRNAs were found to dysregulated in gonadal fat; among them, $68 \%$ of the mRNAs and IncRNAs were downregulated. In the KEGG pathway analysis, most of these RNAs were enriched in metabolic pathways (pyruvate, propanoate, and citrate cycles) and nutrient synthesis (fatty acid, insulin signal, and terpenoid backbones), and the genes involved were all downregulated (Suppl. Table 2). Similarly, the most changed mRNAs and lncRNAs (FC $\geq 2.5$ ) were also downregulated.

Combining the analyses of the placenta and adipose tissue, the different expression tendencies (upregulation in the placenta and downregulation in the adipose tissue) might indicate the different effects of HFD on placenta and adipose tissue during pregnancy. The results indicated that HFD or overnutrition during pregnancy increased the expression of the genes involved in ECM interactions and metabolic pathways, might promote placental development, and ultimately lead to an overgrowth of the foetus. Many studies using an obesogenic diet before or during gestation have demonstrated that a high-sugar diet or HFD increases the expression of glucose and amino acid transporters in the placenta and ultimately induces foetal growth [27] [28-30]. However, our results did not reveal any glucose or amino acid transporter proteins to be differentially expressed but indicated increased expression of genes involved in ECM interactions and metabolic pathways, may indicate nutrients from the maternal diet might not directly influence the RNA levels of nutrient transporter genes in the placenta but promote placental development and ultimately lead to foetal growth.

It is known that nutrient overload leads to lipid accumulation in adiposity, an effect accompanied by increases in fatty acid syntheses and exacerbated insulin resistance. Surprisingly, administration of HFD during pregnancy downregulated the genes enriched in metabolic pathways of gonadal fat, such as the pyruvate, propanoate, and citrate cycle pathways, as well as fatty acid and terpenoid backbone biosynthesis. These findings suggest that the efficiency of energy intake and nutrient synthesis was decreased, which contradicts the phenotype of obese or T2DM mice fed an HFD [31, 32]. Similarly, the genes enriched in insulin signalling were also decreased, which might suggest that adipose tissue presents reduced insulin sensitivity. Combined with the result obtained for placenta, we speculated that some hormones or cell factors secreted from the placenta might suppress energy metabolism, nutrient uptake, and insulin sensitivity in adipose tissue, promote placental development and supply more glucose to foetal growth. The results obtained for the placenta showed that one of the serpin peptides, serpina3n, was significantly increased in HFD mice [33-35]. Serpina3 $\mathrm{n}$ was abnormally highly increased in a Long-Evans agouti T2DM rat model and T2DM patient serum and positively associated with the level of insulin in serum [36], indicating that it might play an important role in energy metabolism and nutrient synthesis in gonadal tissue.

Further analysis identified the 30 most commonly dysregulated genes in placenta and gonadal fat, and pathway analysis indicated that most of these genes were enriched in inflammation (complement and coagulation cascades and chemokine signalling pathway), metabolism (pantothenate and CoA biosynthesis, butanoate metabolism, and nicotinate and nicotinamide metabolism), and vascular growth. In particular, we noted that the pathways of HIF-1 signalling, renin-angiotensin system, and haematopoietic cell lineage were highly associated with vascular endothelial growth factor (VEGF), endometrium/ decidua cell growth, and angiogenesis in late gestation [37]. In human endometrial stromal cells, activation of the renin-angiotensin system is stimulated by the expression of VEGF, PAI-a (serpinde1), Tfrc, and PIK3R1, which activate the placental endometrium toward decidualization and increased placental vascular growth [38], this finding indicates that activation of these pathways might lead to vascular endothelial growth, decidualization of the endometrium, and increased angiogenesis in the placenta.

In conclusion, our study provides potential candidate genes involved in the pathogenesis of GDM. In pregnant mice, HFD upregulated placental gene expression, promoted placental development, and stimulated foetal growth. In parallel, in gonadal fat, HFD downregulated gene expression, suppressed the sensitivity of the insulin signalling and metabolic pathways, provided more nutrients to the placenta, and promoted foetal growth. Taken together with the results of other studies, these findings could advance our understanding of GDM pathogenesis and promote earlier diagnosis, better treatment, and improved outcomes.

\section{KARGER}




\section{Cellular Physiology Cell Physiol Biochem 2018;50:2260-2271

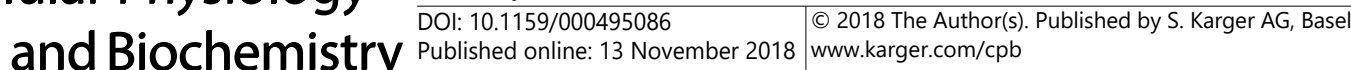

Huang et al.: Profiles of mRNA and IncRNA on Placenta and Fat

\section{Acknowledgements}

This work was supported by the Strategic Priority Research Program of the Chinese Academy of Sciences (Grant No.XDA16020702), NSFC grant (81771611 to JMN, 31671562 to JVZ), the National Major Basic research program of China (2013CB945503 to JVZ), a Guangdong grant (to JVZ), and a Shenzhen grant (JCYJ2017041214026739 to JMN, JCYJ20160331190714896 to JVZ and JCYJ20150403105513698 to LRR).

J.V.Z. and C.H. conceived and designed the experiments; C.H., B.B.H., X.Y.Q. and T.X.X. performed the experiments; C.H, J.M.N., J.C. and L.R.R. analysed the data; Y.Y. and L.R.R. contributed reagents/materials/analysis tools/housing animals; C.H., J.V.Z., and Y.Y. wrote and revised the manuscript.

\section{Disclosure Statement}

Chen Huang, Jian-min Niu, Yan Yu, Bin-bin Huang, Xiao-yun Qin, Tian-xia Xiao, Jie Chen, Li-rong Ren and Jian V. Zhang declare no conflicts of interest.

\section{References}

1 Metzger BE, Lowe LP, Dyer AR, Trimble ER, Chaovarindr U, Coustan DR, Hadden DR, McCance DR, Hod M, McIntyre HD, Oats JJN, Persson B, Rogers MS, Sacks DA, Grp HSCR: Hyperglycemia and adverse pregnancy outcomes. New Engl J Med 2008;358:1991-2002.

- Nolan CJ: Lipotoxicity, beta Cell Dysfunction, and Gestational Diabetes. Cell Metab 2014;19:553-554.

-3 Schwartz ML, Ray WN, Lubarsky SL: The diagnosis and classification of gestational diabetes mellitus: Is it time to change our tune? Am J Obstet Gynecol 1999;180:1560-1569.

-4 Coustan DR: Pregnancy Outcomes in Women With and Without Gestational Diabetes Mellitus According to the International Association of the Diabetes and Pregnancy Study Groups Criteria. Obstet Gynecol 2013;121:377-377.

5 Langer O, Yogev Y, Xenakis EMJ, Brustman L: Overweight and obese in gestational diabetes: The impact on pregnancy outcome. Am J Obstet Gynecol 2005;192:1768-1776.

-6 Jarvie R: Lived experiences of women with co-existing BMI >= 30 and Gestational Diabetes Mellitus. Midwifery 2017;49:79-86.

7 Weijers RNM, Bekedam DJ, Oosting H: The prevalence of type 2 diabetes and gestational diabetes mellitus in an inner city multi-ethnic population. Eur J Epidemiol 1998;14:693-699.

-8 Nahum SK, Friger M, Shoham-Vardi I, Abokaf H, Spiegel E, Sergienko R, Landau D, Sheiner E: Prenatal exposure to gestational diabetes mellitus as an independent risk factor for long-term neuropsychiatric morbidity of the offspring. Am J Obstetrics Gynecol 2016;215:e1-e7.

-9 Chu SY, Callaghan WM, Kim SY, Schmid CH, Lau J, England LJ, Dietz PM: Maternal obesity and risk of gestational diabetes mellitus. Diabetes Care 2007;30:2070-2076.

10 Song L, Sun B, Boersma GJ, Cordner ZA, Yan JQ, Moran TH, Tamashiro KLK: Prenatal high-fat diet alters placental morphology, nutrient transporter expression, and mtorc1 signaling in rat. Obesity 2017;25:909919.

11 Meo SA, Hassain A: Metabolic Physiology in Pregnancy. JPMA J Pak Med Assoc 2016;66:S8-S10.

$\checkmark 12$ Lacroix M, Battista MC, Doyon M, Moreau J, Patenaude J, Guillemette L, Menard J, Ardilouze JL, Perron P, Hivert MF: Higher maternal leptin levels at second trimester are associated with subsequent greater gestational weight gain in late pregnancy. Bmc Pregnancy Childbirth 2016;16:62.

13 Qiao LP, Wattez JS, Lee S, Nguyen A, Schaack J, Hay WW, Shao JH: Adiponectin Deficiency Impairs Maternal Metabolic Adaptation to Pregnancy in Mice. Diabetes 2017;66:1126-1135.

14 Ekinci EI, Torkamani N, Ramchand SK, Churilov L, Sikaris KA, Lu ZX, Houlihan CA: Higher maternal serum prolactin levels are associated with reduced glucose tolerance during pregnancy. J Diabetes Invest 2017;8:697-700.

15 Demirci C, Ernst S, Alvarez-Perez JC, Rosa T, Valle S, Shridhar V, Casinelli GP, Alonso LC, Vasavada RC, GarciaOcana A: Loss of HGF/c-Met Signaling in Pancreatic beta-Cells Leads to Incomplete Maternal beta-Cell Adaptation and Gestational Diabetes Mellitus. Diabetes 2012;61:1143-1152.

16 Al-Badri MR, Zantout MS, Azar ST: The role of adipokines in gestational diabetes mellitus. Ther Adv Endocrinol 2015;6:103-108. 


\section{Cellular Physiology Cell Physiol Biochem 2018;50:2260-2271

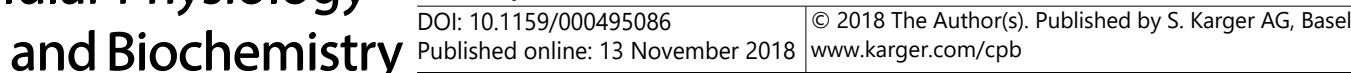

Huang et al.: Profiles of mRNA and IncRNA on Placenta and Fat

17 Hendler I, Blackwell SC, Mehta SH, Whitty JE, Russell E, Sorokin Y, Cotton DB: The levels of leptin, adiponectin, and resistin in normal weight, overweight, and obese pregnant women with and without preeclampsia. Am J Obstet Gynecol 2005;193:979-983.

18 Enquobahrie DA, Williams MA, Qiu CF, Meller M, Sorensen TK: Global placental gene expression in gestational diabetes mellitus. Am J Obstet Gynecol 2009;200:1-13.

19 Li HP, Chen X, Li MQ: Gestational diabetes induces chronic hypoxia stress and excessive inflammatory response in murine placenta. Int J Clin Exp Patho 2013;6:650-659.

20 Radaelli T, Varastehpour A, Catalano P, Haugeul-de Mouzon S: Gestational diabetes induces placental genes for chronic stress and inflammatory pathways. Diabetes 2003;52:2951-2958.

21 Zhao YH, Wang DP, Zhang LL, Zhang F, Wang DM, Zhang WY: Genomic expression profiles of blood and placenta reveal significant immune-related pathways and categories in Chinese women with gestational diabetes mellitus. Diabetic Med 2011;28:237-246.

-22 Reece EA, Leguizamón G, Wiznitzer A, Reece EA, Wiznitzer A: Gestational diabetes: the need for a common ground. Lancet 2009;373:1789-1797.

23 Mestan K, Ouyang F, Matoba N, Pearson C, Ortiz K, Wang X: Maternal obesity, diabetes mellitus and cord blood biomarkers in large-for-gestational age infants. J Pediatr Biochem 2010;1:217-224.

-24 Jones HN, Woollett LA, Barbour N, Prasad PD, Powell TL, Jansson T: High-fat diet before and during pregnancy causes marked up-regulation of placental nutrient transport and fetal overgrowth in C57/BL6 mice. Faseb J 2009;23:271-278.

-25 Rosario FJ, Kanai Y, Powell TL, Jansson T: Increased placental nutrient transport in a novel mouse model of maternal obesity with fetal overgrowth. Obesity 2015;23:1663-1670.

-26 Huang C, Wang MM, Ren LR, Xiang L, Chen J, Li MX, Xiao TX, Ren PG, Xiong LK, Zhang JV: CMKLR1 deficiency influences glucose tolerance and thermogenesis in mice on high fat diet. Biochem Bioph Res Co 2016;473:435441.

-27 Capobianco E, Fornes D, Linenberg I, Powell TL, Jansson T, Jawerbaum A: A novel rat model of gestational diabetes induced by intrauterine programming is associated with alterations in placental signaling and fetal overgrowth. Mol Cell Endocrinol 2016;422:221-232.

28 Carbillon L: Fetal/placental weight ratio in a mouse model of maternal diet-induced obesity. P Natl Acad Sci USA 2016;113:e260-e260.

29 Musial B, Vaughan OR, Fernandez-Twinn DS, Voshol P, Ozanne SE, Fowden AL, Sferruzzi-Perri AN: A Westernstyle obesogenic diet alters maternal metabolic physiology with consequences for fetal nutrient acquisition in mice. J Physiol-London 2017;595:4875-4892.

-30 Sferruzzi-Perri AN, Vaughan OR, Haro M, Cooper WN, Musial B, Charalambous M, Pestana D, Ayyar S, Ferguson-Smith AC, Burton GJ, Constancia M, Fowden AL: An obesogenic diet during mouse pregnancy modifies maternal nutrient partitioning and the fetal growth trajectory. Faseb J 2013;27:3928-3937.

-31 Ramdhave AS, Ojha S, Nandave M: Energy intake correlates with the levels of fatty acid synthase and insulinlike growth factor-1 in male and female C57BL/6 mice. Am J Transl Res 2017;9:830.

-32 Chu DT, Malinowska E, Jura M, Kozak LP: C57BL/6J mice as a polygenic developmental model of diet-induced obesity. Physiol Rep 2017;5:e13093.

33 Hsu I, Parkinson LG, Shen Y, Toro A, Brown T, Zhao H, Bleackley RC, Granville DJ: Serpina3n accelerates tissue repair in a diabetic mouse model of delayed wound healing. Cell Death Dis 2014;5:e1458.

-34 Harriman G, Greenwood J, Bhat S, Huang XY, Wang RY, Paul D, Tong L, Saha AK, Westlin WF, Kapeller R, Harwood HJ: Acetyl-CoA carboxylase inhibition by ND-630 reduces hepatic steatosis, improves insulin sensitivity, and modulates dyslipidemia in rats. P Natl Acad Sci USA 2016;113:e1796-e1805.

35 Boutsikou T, Briana DD, Boutsikou M, Kafalidis G, Stamati L, Baka S, Hassiakos D, Gourgiotis D, MalamitsiPuchner A: Cord blood chemerin and obestatin levels in large for gestational age infants. J Matern-Fetal Neo M 2013;26:123-126.

-36 Takahashi E, Unokikubota H, Shimizu Y, Okamura T, Iwata W, Kajio H, Yamamotohonda R, Shiga T, Yamashita S, Tobe K: Proteomic Analysis of Serum Biomarkers for Pre-Diabetes Using the LEA Rat, a Spontaneous Animal Model of Type 2 Diabetes Mellitus. J Diabetes Invest 2017;8:661-671.

-37 Fryer BH, Simon MC: Hypoxia, HIF and the placenta. Cell Cycle 2006;5:495-498.

-38 Lumbers ER, Wang Y, Delforce SJ, de Meaultsart CC, Logan PC, Mitchell MD, Pringle KG: Decidualisation of human endometrial stromal cells is associated with increased expression and secretion of prorenin. Reprod Biol Endocrin 2015;13:129. 